\title{
La féminisation de la médecine est-elle une réponse à la menace d'une pénurie de médecins?
}

Barbara Weil

Responsable du département Promotion de la santé et prévention de la FMH
Correspondance:

Barbara Weil

FMH

Elfenstrasse 18

CH-3000 Berne 15

barbara.weil@fmh.ch
Dans l'Antiquité et le haut Moyen-Age, il n'était pas coutume pour les femmes d'étudier et d'enseigner dans les académies - mais c'était parfaitement possible: des écrits nous révèlent l'existence de femmes médecins, architectes et autres savantes de renom dès l'Egypte ancienne, la Grèce antique ou l'Empire romain.

La société médiévale obéissait beaucoup plus aux préceptes de l'Eglise. Pendant des siècles, seule une dot versée par la famille à un couvent permettait aux femmes d'accéder à l'éducation. L'étude et l'enseignement de la médecine étaient pour elles l'une des dernières possibilités d'exercer une activité académique.

Le premier à favoriser l'éducation scolaire des jeunes filles a été Jan Amos Coménius, évêque des Frères bohêmes: «Omnes, omnia, omnino» - tous doivent recevoir un enseignement complet sur toutes choses! Mais les femmes ont dû parcourir un chemin long et difficile, jonché d'innombrables obstacles, pour évacuer les préjugés, avant de pouvoir accéder librement à la formation universitaire, et ainsi surmonter le débat qui a perduré des siècles: les femmes possédaient-elles les facultés mentales et physiques nécessaires pour les études?

Aujourd'hui, de telles déclarations prêteraient plutôt à sourire, d'autant plus que les femmes sont depuis longtemps majoritaires dans la filière médicale et que la féminisation de la médecine [1] est désormais en route. Droit de vote, libre choix de la profession, autonomie professionnelle après le mariage, propre compte bancaire - tous ces aspects sont aujourd'hui une évidence, indissociable du quotidien des femmes actuelles. La Réglementation pour la formation postgraduée offre aussi aux femmes la possibilité d'accomplir leur formation spécifique à temps partiel (voir encadré). Vu sous cet angle, la médecine est un domaine idéal pour les femmes, lesquelles

\section{Art. 32 Travail à temps plein et à temps partiel}

- Jusqu'à la moitié de la formation postgraduée spécifique peut être accomplie à temps partiel, à moins que le programme de formation postgraduée n'autorise une part plus importante.

- L'intégralité de la formation postgraduée non spécifique peut être accomplie à temps partiel.

- L'étendue de l'engagement à temps partiel doit correspondre au moins à $50 \%$ d'un emploi à plein temps. La formation postgraduée accomplie à temps partiel doit être prise en compte proportionnellement.

- Pour les titres de spécialiste qui n'exigent pas de formation postgraduée spécifique, le pourcentage de travail partiel admis est réglé dans le programme de formation postgraduée. contribueront notamment à apporter une réponse à la pénurie de médecins qui se profile.

Comparer les possibilités offertes sur le papier avec la réalité actuelle soulève toutefois plusieurs questions auxquelles il faut répondre si l'on souhaite remédier de manière efficace au manque imminent de médecins en Suisse $[2,3,4]$.

- Les possibilités de formation postgraduée et les conditions de travail sont-elles adaptées aux besoins actuels des femmes médecins ou se basentelles encore sur des stéréotypes professionnels et sociétaux traditionnellement masculins?

- Toutes les disciplines proposent-elles des emplois à temps partiel véritablement souples et à tous les échelons hiérarchiques (par ex. médecine interne, chirurgie, orthopédie, etc.)?

- Les crèches internes aux hôpitaux destinées au personnel médical sont aujourd'hui très répandues, et pourtant: existe-t-il suffisamment de crèches (finançables) avec des heures d'ouverture adaptées aux temps de travail des médecins (service du matin, service de nuit)?

- Au regard de la maternité/famille et de la carrière professionnelle, les opinions évoluent-elles ou sont-elles encore prisonnières de «l'incompatibilité d'associer vie de famille et carrière»?

- Des programmes de mentoring suffisamment adaptés sont-ils proposés?

La nouvelle génération de médecins n'est pas prête à faire carrière à tout prix, et encore moins au détriment de sa famille. Les pères souhaitent, eux aussi, s'occuper de leurs enfants. Une véritable égalité entre femmes et hommes est probablement l'une des réponses.

\section{Références}

1 Kraft E, Hersperger M. Le corps médical en Suisse la féminisation de la médecine. Bull Méd Suisses. 2009;90(47):1823-5

2 Buddeberg-Fischer B, Stamm M. The medical profession and young physicians' lifestyles in flux: challenges for specialty training and health care delivery systems. Swiss Med Wkly. 2010;140:w13134.

3 Stamm M, Buddeberg-Fischer B. How do physicians and their partners coordinate their careers and private lives? Swiss Med Wkly. 2011:141w13179.

4 Buddeberg-Fischer B, Stamm M, Buddeberg C, Bauer G, Hämmig O, Knecht M. The impact of gender and parenthood on physicians' careers - professional and personal situation seven years after graduation BMC. Health Services Research. 2010;10(40). 